\title{
Genome Sequencing and Analysis Reveal the Mechanism of Terpenoids Biosynthesis in Deep- See-Derived Fungus Aspergillus Tubingensis
}

Shan Lin

Beijing University of Chinese Medicine

Shiping Hu

Beijing University of Chinese Medicine

Zhenyun Han

Beijing University of Chinese Medicine

Xiaoni Chen

Beijing University of Chinese Medicine

Xueying Lu

Beijing University of Chinese Medicine

Fenfang Wu (D wufenfang99@yahoo.com)

Beijing University of Chinese Medicine

Original article

Keywords: Deep-sea fungus, Aspergillus tubingensis, terpenoid, biosynthesis

Posted Date: June 1st, 2021

DOl: https://doi.org/10.21203/rs.3.rs-553748/v1

License: (1) This work is licensed under a Creative Commons Attribution 4.0 International License.

Read Full License 


\section{Abstract}

In recent years, deep-sea-derived fungi have become an important source of marine natural products. Aspergillus tubingensis is extremely rich in secondary metabolites, which is a valuable deepsea-derived fungus needs to be further explored. So far, many small molecular compounds with novel structure and significant biological activity have been discovered from A. tubingensis, among which terpenoids account for about $20 \%$, showing great research potential in anti-tumor, antiviral and antibacteria. Although more and more new terpenoids have been discovered from A. tubingensis and their structures have been identified, few studies have investigated the biosynthetic pathway of terpenoid. In order to further elucidate the mechanism of terpenoid biosynthesis, the key genes and enzymes involved in terpenoid biosynthesis were successfully mined and further analyzed based on genome sequencing analysis. Subsequently, hydroxymethylglutaryl-CoA synthase, mevalonate kinase, phosphomevalonate kinase as well as diphosphomevalonate decarboxylase were annotated, which played important roles in terpenoid biosynthesis of $A$. tubingensis. Furthermore, the biosynthetic pathway of terpenoid in $A$. tubingensis has been constructed, which could be applied to develop the metabolic regulation of $A$. tubingensis. This study would provide more sufficient scientific basis and new ideas for the genetic regulation of terpenoid biosynthesis in A. tubingensis.

\section{Introduction}

The deep sea is one of the most mysterious and unexplored extreme environments in the world, characterized by lack of light, low temperatures, anaerobic conditions, and hydrostatic pressures that could reach more than 1000 atmospheres (Thornburg et al. 2010). Over the past 50 years, there have been about 24,000 natural products of marine origin, but less than $2 \%$ of them come from deep-sea sources. Generally, deep-sea microorganisms have unique physiological metabolic processes by the adaptation to extreme environmental conditions, and thus exhibit the chemical structure diversity, novelty, and significant biological activity of their metabolites (Skropeta and Wei 2014). It has been reported that over $75 \%$ of natural products from deep-sea sources are bioactive, and more than half show significant cytotoxic activity against human tumor cell lines (Skropeta 2008). As an important group of deep-sea microorganisms, deep-sea fungi are well known for their diversity of secondary metabolites, which have the potential to treat human diseases and find new biological targets, providing important support for marine drug research and development (Xu et al. 2018).

Aspergillus tubingensis, as a valuable deep-sea-derived fungus needs to be further explored, is extremely rich in secondary metabolites (Koch et al. 2014). At present, anthraquinones, alkaloids, terpenoids and other compounds have been isolated from A. tubingensis, and many of the secondary metabolites have the activities of antibacterial, anti-inflammatory, anti-cancer and antiviral, which have a certain potential as drugs (Carboue et al. 2019; Ottoni et al. 2019).

Terpenoids refer to derivatives with $\left(\mathrm{C}_{5} \mathrm{H}_{8}\right)_{\mathrm{n}}$ general formula, oxygen content and different saturation levels, which can be regarded as a class of natural compounds linked in various ways by isoprene or 
isopentane (Hillier and Lathe 2019). Although terpenoids are not the dominant components in $A$. tubingensis, they play an important role among the active ingredients because of their anti-tumor, antiviral and anti-inflammatory activities. It was reported that five terpenoids of 20-norisopimarane were isolated from Aspergillus from deep-sea sediments in the South China Sea, which showed significant antibacterial activity against Fusarium graminearum (Li et al. 2016). Furthermore, a new indole diterpene Penicindopene A was isolated from Penicillium YPCMAC1 from deep sea of western pacific, which showed moderate cytotoxicity to A549 and HeLa cell lines (Liu et al. 2019). Subsequently, two unique phenol-sesquiterpenoids, phomeroid A-B, were isolated from Phomopsis tersa FS441 from deep-sea sediment samples in the Indian Ocean, which showed moderate inhibitory activity against human glioblastoma SF-268, breast cancer MCF-7, liver cancer HepG-2 and lung cancer A549 (Chen et al. 2020). The above studies indicated that more and more novel chemical structures derived from deep-sea microorganisms have been discovered, with the development of deep-sea microbial sample collection, separation and purification technology and compound structure analysis technology, which is beneficial to the excavation of deep-sea fungal resources (Li et al. 2015).

Even so, few studies have investigated the biosynthetic pathway of terpenoid, and it is becoming more and more urgent to investigate the hereditary information or functional genes of $A$. tubingensis by omics sequencing technology (Lin et al. 2019). Therefore, the functional genes involved in the biosynthetic pathway can be obtained by genome sequencing analysis, and the biological processes in different states can be revealed (Xiong and Zhao 2018). In the present study, to better understand the molecular factors and their regulatory genes involved in accumulation of terpenoid, the genomic profiles of $A$. tubingensis were analyzed. We gain insights into the terpenoid accumulation mechanism of $A$. tubingensis, particularly the functional genes and enzymes involved in terpenoid biosynthesis. Physiological observations such as growth and terpenoid biosynthesis were linked to genomic data obtained by genome sequencing. These results would provide novel insight into understanding the molecular mechanisms of terpenoid accumulation and aid in understanding its biosynthesis, and developing future studies on the metabolic regulation of $A$. tubingensis.

\section{Materials And Methods}

\section{Strains and genomic data}

The Draft Genome Sequence of $A$. tubingensis WU-2223L, a Citric Acid-producing Filamentous Fungus Belonging to Aspergillus Section Nigri, has been submitted to NCBI (BLWE00000000). The Genome Coverage was $163 x$, which was applied by HiSeq X; Nanopore MinION. The Assembly Method was MaSuRCA v. 3.3.3. The genomic profiles of $A$. tubingensis were downloaded for further bioinformatics analysis.

\section{Functional gene mining of terpenoid biosynthesis in A. tubingensis}

The files of genome sequencing results were downloaded on computer. Subsequently, the annotation result files of assembled Unigenes from the genome sequencing results were obtained. Noteworthy, 
paired-end reads were used again for gap filling of scaffolds to obtain sequences with least Ns and cannot be extended on either end. Such sequences were defined as Unigenes. Unigene annotation provides information of expression and functional annotation of Unigene. The "annotation files" path was opened, and "map 00900" in the search bar was entered. Then, terpenoid backbone biosynthesis (map00900) in the KEGG classification of annotation files was searched. Furthermore, the functional genes such as hydroxymethylglutaryl-CoA synthase, mevalonate kinase, phosphomevalonate kinase and diphosphomevalonate decarboxylase, were mined and analyzed to confirm whether annotated in $A$. tubingensis genome.

\section{Bioinformatic analysis of functional genes involved in terpenoid biosynthesis}

The fungus $A$. tubingensis, along with the annotation results of Unigenes, which has been submitted on NCBI (BLWE00000000). Open reading frame (ORF) of functional genes was analyzed by ORF program (http://www.ncbi.nlm.nih.gov/gorf/gorf.html). Theoretical molecular mass and isoelectric point of functional enzymes was calculated by ProtParam tool (http://us.expasy.org/tools/protparam.html), and catalytic domain was identified by InterProScan (http://www.ebi.ac.uk/Tools/InterProScan/). Moreover, protein secondary structure was predicted by Predict Protein (http://www.predictprotein.org/) online. In addition, the amino acid sequences of 18SRNA from different fungi were aligned using the Clustal $X$ program (http://www.clustal.org/). Finally, phylogenetic tree was carried out by MEGA 4.0 (https://www.megasoftware.net/mega4/).

\section{Construction of biosynthetic pathway of terpenoid in A. tubingensis}

Based on the KEGG Pathway annotation information by genome sequencing and short read sequence assembly, the biosynthetic pathways of terpenoid in A. tubingensis were analyzed. The metabolic pathways of terpenoid were searched and screened from KEGG PATHWAY Database (http://www.genome.jp/kegg/pathway.html). Subsequently, the KEGG Pathway annotation Database was used to find the annotation pathways corresponding to the Pathway numbers obtained from the KEGG Pathway Database. The online KEGG Pathway database and genomic KEGG Pathway annotation information were compared and analyzed to study the biosynthetic pathways of terpenoids, as well as the corresponding Unigene sequences of related enzymes.

\section{Results}

\section{Phylogenetic tree analysis of 18S rRNA}

The deep-sea fungi have the characteristics of species diversity. Phylogenetic tree analysis of the genetic relationships could be carried out to classify the characteristics at a higher level based on molecular biology and ITS region sequencing. The phylogenetic tree of $A$. tubingensis and other strains in Genbank was shown in Fig. 1, which indicated that $A$. tubingensis is closely related to Aspergillus magaliesburgensis (MK450649.1) and Aspergillus alliaceus (EF661548.1) in evolutionary relationship, 
forming a relatively independent branch. These diverse species of deep-sea fungi provided abundant secondary metabolites for humans.

\section{Draft genome sequence of $A$. tubingensis}

The A. tubingensis whole genome shotgun (WGS) project has the project accession BLWE00000000, which has been submitted to NCBI(Yoshioka et al. 2020). This version of the project has the accession number BLWE01000000, and consists of sequences BLWE01000001-BLWE01000015. We downloaded the genomic profiles from NCBI for further bioinformatics analysis, and the genome features of $A$. tubingensis were shown in Table 1.

Table 1

Genome features of $A$. tubingensis

\begin{tabular}{|ll|}
\hline Characteristics & A.tubingensis \\
\hline Genome Coverage & $163 \mathrm{x}$ \\
\hline Contigs & 15 \\
\hline Proteins & 11,479 \\
\hline Total length & $35,047,229 \mathrm{bp}$ \\
\hline BioProject & PRJDB9560 \\
\hline BioSample & SAMD00216538 \\
\hline
\end{tabular}

Mining functional proteins involved in terpenoid biosynthesis of A. tubingensis

Paired-end reads were used for gap filling of scaffolds to obtain Unigenes with least Ns that cannot be extended on either end. Furthermore, the files of annotation result of assembled Unigenes from the genome sequencing were obtained for further analysis. Subsequently, the functional proteins involved in terpenoid biosynthesis of $A$. tubingensis were shown in Table 2. Among them, hydroxymethylglutaryl-CoA synthase, mevalonate kinase, phosphomevalonate kinase as well as diphosphomevalonate decarboxylase were annotated, which played important roles in terpenoid biosynthesis of $A$. tubingensis. 
Table 2

Functional proteins involved in terpenoid biosynthesis of $A$. tubingensis

\begin{tabular}{|lllll|}
\hline Protein Name & $\begin{array}{l}\text { Protein } \\
\text { Accession }\end{array}$ & $\begin{array}{l}\text { Protein } \\
\text { Length }\end{array}$ & $\begin{array}{l}\text { Molecular } \\
\text { weight }\end{array}$ & $\begin{array}{l}\text { Theoretical } \\
\text { pl }\end{array}$ \\
\hline $\begin{array}{l}\text { hydroxymethylglutaryl-CoA } \\
\text { synthase }\end{array}$ & GFN16044.1 & 460 & 50832.65 & 5.98 \\
\cline { 2 - 3 } & GFN17970.1 & 451 & 49879.84 & 5.72 \\
\hline mevalonate kinase & GFN12941.1 & 539 & 58477.35 & 6.77 \\
\hline $\begin{array}{l}\text { phosphomevalonate kinase } \\
\text { diphosphomevalonate }\end{array}$ & GFN11149.1 & 463 & 50637.27 & 5.21 \\
\hline $\begin{array}{l}\text { decarboxylase } \\
\text { GFN12862.1 }\end{array}$ & 762 & 82702.22 & 8.98 \\
\hline
\end{tabular}

Subsequently, bioinformatics analysis of the above proteins was developed, and the molecular weight and theoretical pl were shown in Table 2, respectively. Among them, diphosphomevalonate decarboxylase (GFN12862.1) was alkaline protein, mevalonate kinase (GFN12941.1) was neutral protein, and the rest were acidic proteins.

\section{Secondary and tertiary structures of functional proteins involved in terpenoid biosynthesis}

In order to further understand the function of these proteins, secondary and tertiary structures of the functional proteins involved in terpenoid biosynthesis of $A$. tubingensis were predicted. The predicted secondary structure composition of functional proteins was shown in Table 3, which showed the ratio of strand to helix in each protein, respectively. Subsequently, the predicted tertiary structures of functional proteins involved in terpenoid biosynthesis were shown in Fig. 2, which could indicate the protein family to which the protein belongs and the preference of the substrate.

Table 3

Predicted secondary structure composition of functional proteins involved in terpenoid biosynthesis of $A$. tubingensis.

\begin{tabular}{|lllll|}
\hline Protein Name & Protein Accession & Helix (\%) & Strand (\%) & Loop (\%) \\
\hline hydroxymethylglutaryl-CoA synthase & GFN16044.1 & 41.52 & 13.26 & 45.22 \\
\cline { 2 - 5 } & GFN17970.1 & 43.33 & 13.11 & 43.56 \\
\hline mevalonate kinase & GFN12941.1 & 26.16 & 15.77 & 58.07 \\
\hline phosphomevalonate kinase & GFN11149.1 & 36.50 & 19.01 & 44.49 \\
\hline diphosphomevalonate decarboxylase & GFN12862.1 & 12.86 & 0.66 & 86.48 \\
\cline { 2 - 5 } & GFN12877.1 & 25.50 & 13.86 & 60.64 \\
\cline { 2 - 5 } & & & & \\
\hline
\end{tabular}

Constructing the predicted biosynthetic pathway of terpenoid in A. tubingensis 
Subsequently, terpenoid backbone biosynthesis (map00900) was applied to construct the biosynthetic pathway of terpenoid in $A$. tubingensis. The functional proteins involved in terpenoid biosynthesis of $A$. tubingensis were shown, as well as the predicted biosynthetic pathway of terpenoid in A. tubingensis was shown in Fig. 3, which might provide more sufficient scientific basis and new ideas for the genetic regulation of terpenoid biosynthesis in A. tubingensis. In this pathway, the biosynthesis up to terpenoid in A. tubingensis is different from that in other organisms. Following acetyl-CoA was converted to 3hydroxy-3-methyl-glutaryl-CoA by hydroxymethylglutaryl-CoA synthase, and 3-hydroxy-3-methyl-glutarylCoA was converted to mevalonate by hydroxymethylglutaryl-CoA reductase, mevalonate was subsequently converted to mevalonate- $5 \mathrm{P}$ by mevalonate kinase, and mevalonate- $5 \mathrm{P}$ was further converted to mevalonate-5PP by phosphomevalonate kinase. Moreover, mevalonate-5PP was converted to isopentenyl-PP by diphosphomevalonate decarboxylase, and isopentenyl-PP was used as precusor

substance for monoterpenoid biosynthesis, diterpenoid biosynthesis and carotenoid biosynthesis and so on.

\section{Discussion}

Deep-sea environment is an extreme environment characterized by high pressure, low oxygen, high salt and no illumination. In order to adapt to the survival and reproduction in the deep-sea environment, deepsea fungi have evolved unique physiological structure and metabolic pathway, and can produce novel structure and significant variety of secondary metabolites with biological activities, which become an important resource for the development of new drugs (Zain UI Arifeen et al. 2019). However, limited sampling techniques have limited the study of deep-sea fungi and their metabolites (Daletos et al. 2018). In addition, there are hitherto unknown fungal lineages in the deep sea, which have unique ecological and physiological characteristics, and are difficult to grow through conventional culture methods. Fortunately, with the rapid development of deep-sea sampling and multiomics, abundant active natural products will be mined and contribute to the sustainable use of marine microbial resources and drug development. Therefore, it is more and more urgent to investigate the active ingredients of deep-sea fungi.

There are several studies focused on bacteria and archaea in deep sea, involving a wide range of species, including population distribution, separation of new active metabolites and so on (Carroll et al. 2021; Shin 2020). As marine eukaryotic microorganisms, marine fungi are the main decomposers of low organic matter, which are characterized by diverse species, wide distribution and complex metabolic pathways. In recent years, more than half of the new compounds derived from marine microbes were come from marine fungi (Carroll et al. 2021; Shin 2020). Secondary metabolites isolated from marine fungi have been found to have many important biological activities, such as anti-tumor, antibacterial, antioxidant, antiviral, anti-adhesion and so on (Moghadamtousi et al. 2015). Although terpenoids account for about $20 \%$ of the total active ingredients in A. tubingensis, they play an important role because of their antitumor, antiviral and anti-inflammatory activities (Khizar et al. 2020). Unfortunately, few studies have investigated the biosynthetic pathway of terpenoid, and the hereditary information or functional genes of A. tubingensis by omics sequencing technology is becoming more and more urgent to be investigated. In this study, we investigated the functional genes as well as proteins involved in the biosynthetic pathway 
of terpenoid by omics sequencing technology for the first time, which could gain insights into the terpenoid accumulation mechanism of $A$. tubingensis, particularly the functional genes in terpenoid biosynthesis.

So far, there were several studies investigating the pharmacological effects of terpenoid. However, the studies on the functional genes as well as the enzymes involved in terpenoid biosynthesis of deep-sea fungi were few. For instance, the fungal terpenoid biosynthesis from biochemical, genetic, and genomic viewpoints were investigated, as well as the enzymes involved in synthesizing, transferring, and cyclizing the prenyl chains were systematically discussed, which provided the genomic information and functional evidence for biosynthetic mechanism of terpenoid (Schmidt-Dannert 2015). Furthermore, the characterization and evolution of gene clusters for terpenoid phytoalexin biosynthesis in tobacco were investigated, 1181 metabolic gene clusters with 34 of them potentially being involved in terpenoid biosynthesis were identified, which demonstrated that phytoalexins in tobacco can arise from operon-like gene clusters (Chen et al. 2019). Unfortunately, the key genes involved in terpenoid biosynthesis of deepsea fungi were lacking in-depth genomic mining and analysis. Therefore, it is certainly needed to conduct the study of genome analysis of the key genes and enzymes involved in terpenoid biosynthesis of deepsea fungi.

To our knowledge, genome sequencing and analysis could provide information of gene expression and infer the gene functions (Liu et al. 2015; Wu et al. 2020). Next-generation sequencing technology of genome could systematically provide a complete view of expressed genes and their expression levels (Gu et al. 2019). The availability of a draft genome sequence opens new avenues for new exploration, application and improvements of marine fungi (Kumar et al. 2018). It will lead to the identification and manipulation of candidate genes or genomic regions to generate the new ways to synthesize new compounds with potentials in pharmaceuticals (Wang et al. 2010). In order to obtain a comprehensive insight into the biosynthesis of terpenoid in A. tubingensis, functional genes screened from the submitted genome database of $A$. tubingensis on $\mathrm{NCBI}$, and their evolutionary relationships has been demonstrated. The availability of genome will facilitate the development of new products, and improve the efficient processes of production. The work present here would aid in understanding and carrying out future research on the genetic basis of biology of this organism and contribute to the further production and application of $A$. tubingensis.

\section{Declarations}

\section{Acknowledgements}

Not applicable.

\section{Authors' contributions}

SL, SPH and FFW conceived the study. SL, ZYH, XNC and FFW designed and performed most of the experiments. SL wrote the manuscript; SL, SPH, ZYH, XNC, XYL and FFW edited the manuscript. All 
authors read and approved the final manuscript.

\section{Funding}

This work was financially supported by the National Natural Science Foundation of China (81803652, $31871244,81973733)$, Natural Science Foundation of Guangdong Province (2018A0303100007, 2019A1515011555), Shenzhen Foundation of Health and Family Planning Commission (SZBC2018016), Special Fund for Economic and Technological Development of Longgang District of Shenzhen City (LGKCYLWS2020001571, LGKCYLWS2019000361).

\section{Availability of data and materials}

The data supporting the conclusions are presented in the main article

\section{Ethics approval and consent to participate}

Not applicable.

\section{Consent for publication}

Not applicable.

\section{Conflict of Interest}

The authors declare no conflict of interest.

\section{References}

Carboue Q, Maresca M, Herbette G, Roussos S, Hamrouni R, Bombarda I (2019) Naphtho-gamma-pyrones produced by Aspergillus tubingensis G131: New source of natural nontoxic nntioxidants. Biomolecules 10(1) 29.

Carroll AR, Copp BR, Davis RA, Keyzers RA, Prinsep MR (2021) Marine natural products. Natural product reports 38(2):362-413.

Chen S, Liu Z, Tan H, Chen Y, Li S, Li H, Zhu S, Liu H, Zhang W (2020) Phomeroids A and B: two novel cytotoxic meroterpenoids from the deep-sea-derived fungus Phomopsis tersa FS441. Organic Chemistry Frontiers 7(3):557-562

Chen X, Liu F, Liu L, Qiu J, Fang D, Wang W, Zhang X, Ye C, Timko MP, Zhu QH, Fan L, Xiao B (2019) Characterization and evolution of gene clusters for terpenoid phytoalexin biosynthesis in tobacco. Planta 250(5):1687-1702.

Daletos G, Ebrahim W, Ancheeva E, El-Neketi M, Song W, Lin W, Proksch P (2018) Natural products from deep-sea-derived fungi-A new source of novel bioactive compounds? Current medicinal chemistry 
Gu W, Miller S, Chiu CY (2019) Clinical metagenomic next-generation sequencing for pathogen detection. Annual review of pathology 14:319-338.

Hillier SG, Lathe R (2019) Terpenes, hormones and life: isoprene rule revisited. The Journal of endocrinology 242(2):R9-R22.

Khizar M, Shi J, Saleem S, Liaquat F, Ashraf M, Latif S, Haroon U, Hassan SW, Rehman SU, Chaudhary HJ, Quraishi UM, Munis MFH (2020) Resistance associated metabolite profiling of Aspergillus leaf spot in cotton through non-targeted metabolomics. PloS one 15(2):e0228675.

Koch L, Lodin A, Herold I, llan M, Carmeli S, Yarden O (2014) Sensitivity of neurospora crassa to a marinederived Aspergillus tubingensis anhydride exhibiting antifungal activity that is mediated by the MAS1 protein. Marine drugs 12(9):4713-31.

Kumar A, Sorensen JL, Hansen FT, Arvas M, Syed MF, Hassan L, Benz JP, Record E, Henrissat B, Poggeler $S$, Kempken $F(2018)$ Genome sequencing and analyses of two marine fungi from the north sea unraveled a plethora of novel biosynthetic gene clusters. Scientific reports 8(1):10187.

Li K, Chung-Davidson YW, Bussy U, Li W (2015) Recent advances and applications of experimental technologies in marine natural product research. Marine drugs 13(5):2694-713.

Li XD, Li XM, Li X, Xu GM, Liu Y, Wang BG (2016) Aspewentins D-H, 20-Nor-isopimarane derivatives from the deep sea sediment-derived fungus Aspergillus wentii SD-310. Journal of natural products 79(5):134753.

Lin S, Zou Z, Zhou C, Zhang H, Cai Z (2019) Transcriptome analysis reveals the molecular mechanisms underlying adenosine biosynthesis in anamorph strain of caterpillar fungus. BioMed research international 2019:1864168.

Liu L, Xu W, Li S, Chen M, Cheng Y, Yuan W, Cheng Z, Li Q (2019) Penicindopene A, a new indole diterpene from the deep-sea fungus Penicillium sp. YPCMAC1. Natural product research 33(20):2988-2994.

Liu ZQ, Lin S, Baker PJ, Wu LF, Wang XR, Wu H, Xu F, Wang HY, Brathwaite ME, Zheng YG (2015) Transcriptome sequencing and analysis of the entomopathogenic fungus Hirsutella sinensis isolated from Ophiocordyceps sinensis. BMC genomics 16:106.

Moghadamtousi SZ, Nikzad S, Kadir HA, Abubakar S, Zandi K (2015) Potential antiviral agents from marine fungi: An overview. Marine drugs 13(7):4520-38.

Ottoni CA, Maria DA, Goncalves P, de Araujo WL, de Souza AO (2019) Biogenic Aspergillus tubingensis silver nanoparticles' in vitro effects on human umbilical vein endothelial cells, normal human fibroblasts, HEPG2, and Galleria mellonella. Toxicology research 8(6):789-801. 
Schmidt-Dannert C (2015) Biosynthesis of terpenoid natural products in fungi. Advances in biochemical engineering/biotechnology 148:19-61.

Shin HJ (2020) Natural Products from Marine Fungi. Marine drugs 18(5) 230.

Skropeta D (2008) Deep-sea natural products. Natural product reports 25(6):1131-66.

Skropeta D, Wei L (2014) Recent advances in deep-sea natural products. Natural product reports 31(8):999-1025.

Thornburg CC, Zabriskie TM, McPhail KL (2010) Deep-sea hydrothermal vents: potential hot spots for natural products discovery? Journal of natural products 73(3):489-99.

Wang M, Liu S, Li Y, Xu R, Lu C, Shen Y (2010) Protoplast mutation and genome shuffling induce the endophytic fungus Tubercularia sp. TF5 to produce new compounds. Current microbiology 61(4):254-60.

Wu F, Hu S, Ran Y, Chen X, Lin S (2020) Gene mining and sequence analysis of purine nucleosidase based on RNA-Seq. Journal of visualized experiments : JoVE(164).

Xiong J, Zhao WL (2018) Advances in multiple omics of natural-killer/T cell lymphoma. Journal of hematology \& oncology 11(1):134.

Xu JL, Liu HX, Chen YC, Tan HB, Guo H, Xu LQ, Li SN, Huang ZL, Li HH, Gao XX, Zhang WM (2018) Highly substituted benzophenone aldehydes and eremophilane derivatives from the deep-sea derived fungus Phomopsis lithocarpus FS508. Marine drugs 16(9) 329.

Yoshioka I, Takahashi H, Kusuya Y, Yaguchi T, Kirimura K (2020) Draft genome sequence of Aspergillus tubingensis WU-2223L, a citric acid-producing filamentous fungus belonging to Aspergillus section nigri. Microbiology resource announcements 9(33) e00702-20.

Zain UI Arifeen M, Ma YN, Xue YR, Liu CH (2019) Deep-sea fungi could be the new arsenal for bioactive molecules. Marine drugs 18(1) 9.

\section{Figures}




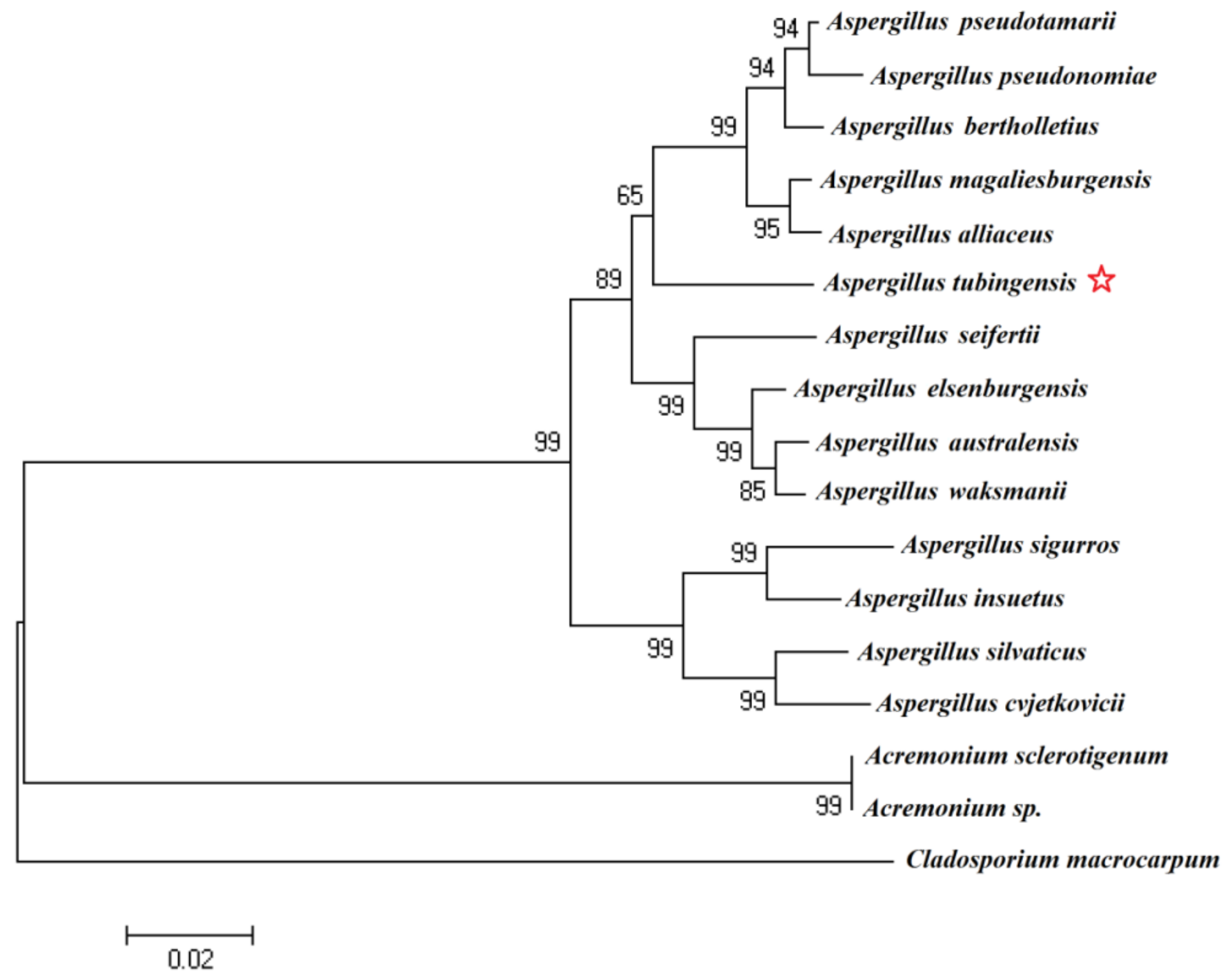

Figure 1

Phylogenetic tree of $18 \mathrm{~S}$ rRNA of A. tubingensis. 


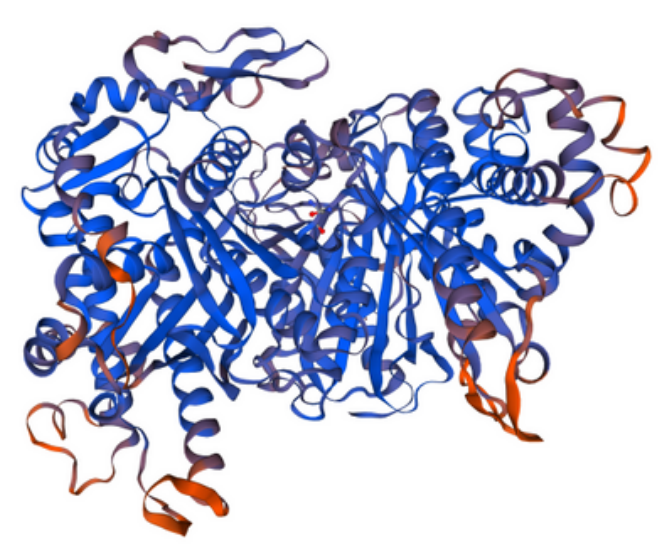

A

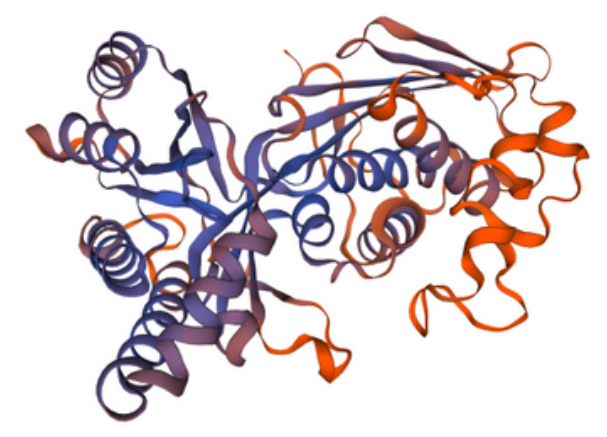

C

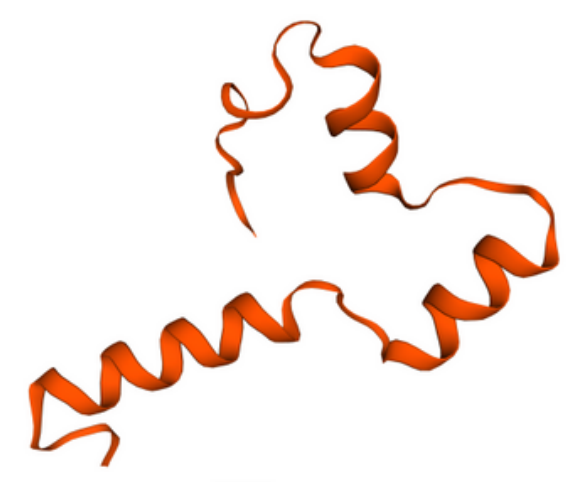

$\mathbf{E}$

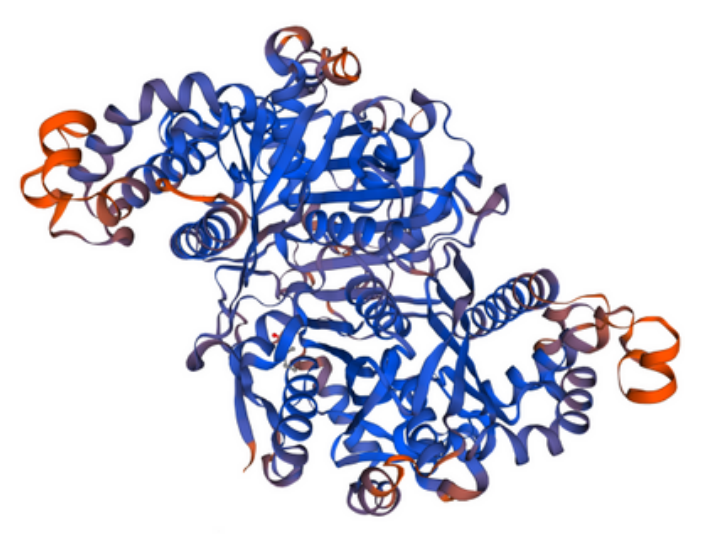

B
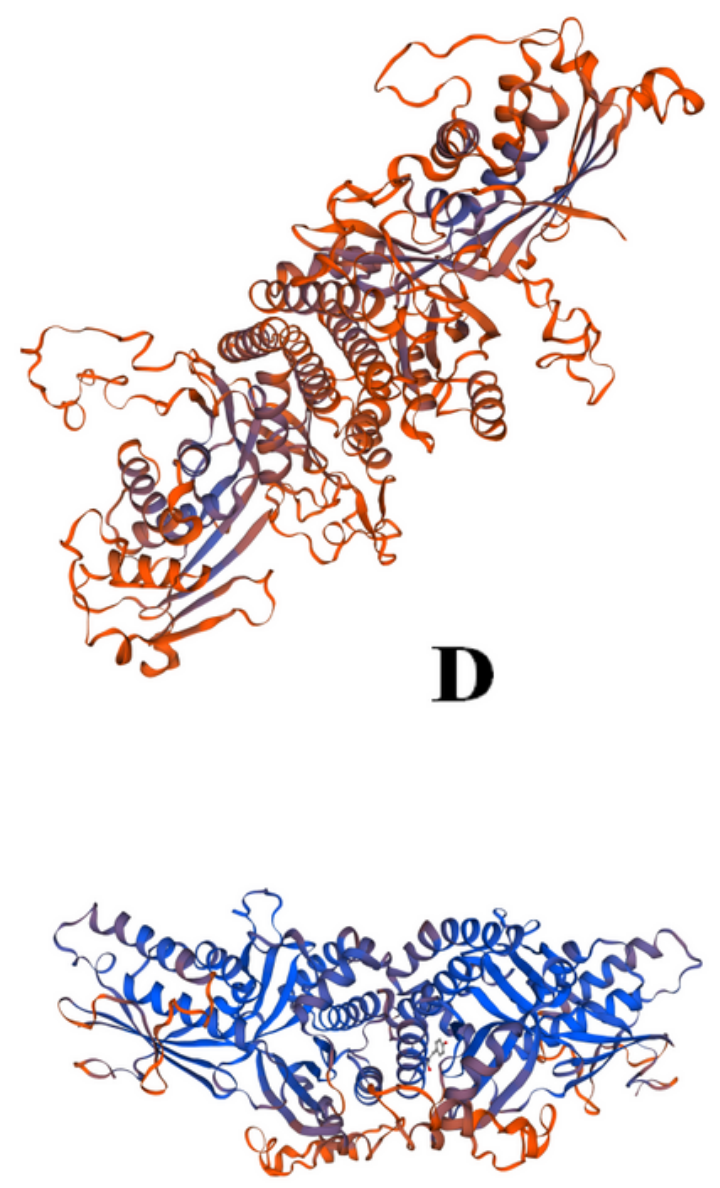

$\mathbf{F}$

\section{Figure 2}

The predicted tertiary structures of functional proteins involved in terpenoid biosynthesis. (A). Hydroxymethylglutaryl-CoA synthase: GFN16044.1; (B). Hydroxymethylglutaryl-CoA synthase: GFN17970.1; (C). Mevalonate kinase: GFN12941.1; (D). Phosphomevalonate kinase: GFN11149.1; (E). Diphosphomevalonate decarboxylase: GFN12862.1; (F). Diphosphomevalonate decarboxylase: GFN12877.1. 


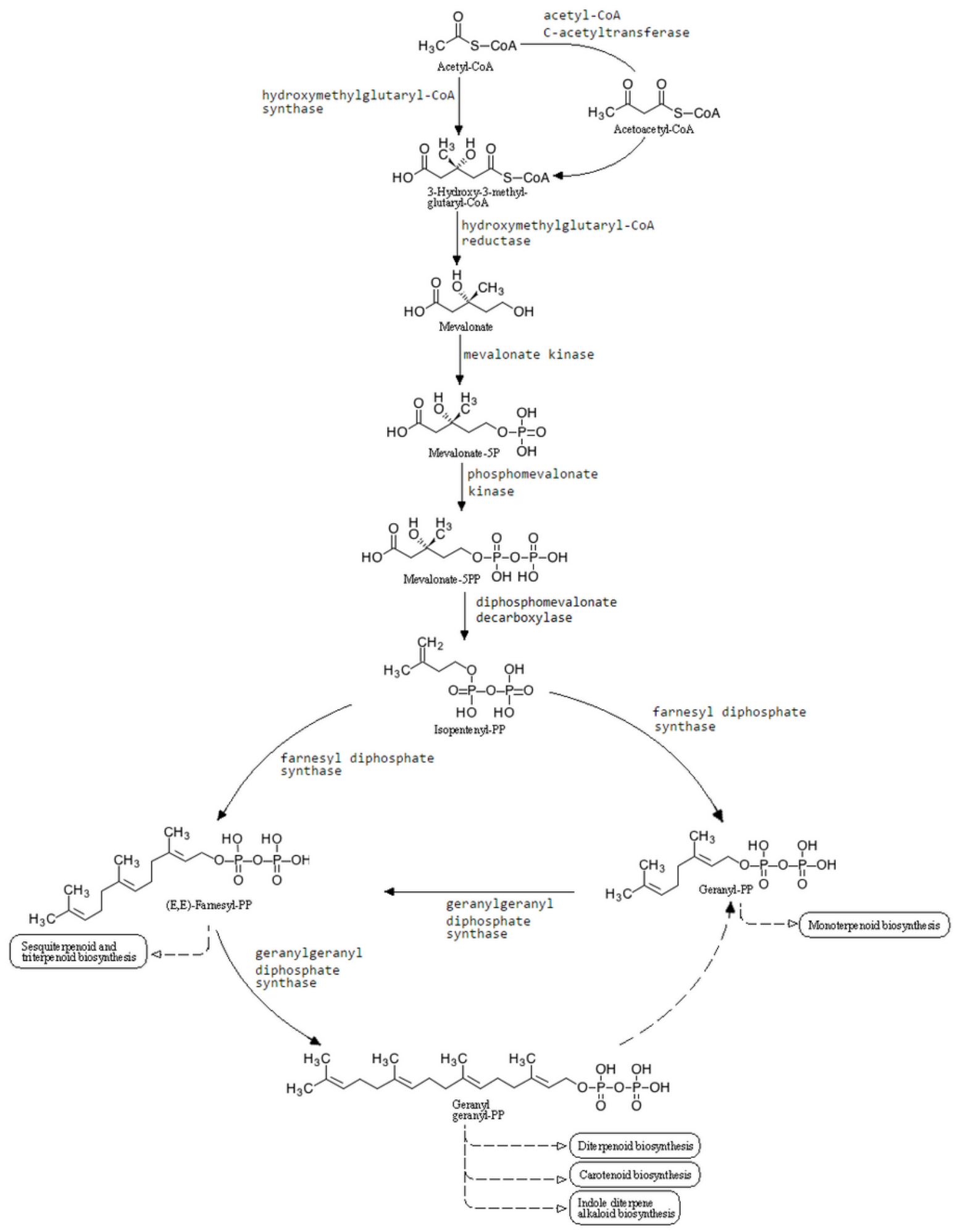

Figure 3

The predicted biosynthetic pathway of terpenoid in A. tubingensis. 